\title{
Local well-posedness for periodic Benjamin equation with small initial data
}

\author{
Shaoguang Shi ${ }^{{ }^{*}}$ and Junfeng $\mathrm{Li}^{2,3}$
}

"Correspondence:

shishaoguang@mail.bnu.edu.cn

${ }^{1}$ Department of Mathematics, Linyi University, Linyi, 276005, P.R. China

Full list of author information is

available at the end of the article

\begin{abstract}
The local well-posedness of the periodic Benjamin equation with small initial value in $H^{5}(\mathbb{T}), s \geq-1 / 2$, is given. It is here shown that $-1 / 2$ is the lower endpoint to obtain the bilinear estimates which are the crucial steps to obtain the local well-posedness by the Picard iteration.
\end{abstract}

MSC: 35Q53; 35Q55

Keywords: Benjamin equation; local well-posedness; bilinear estimate

\section{Introduction}

The initial value problem (IVP) associated to the periodic Benjamin equation is

$$
\left\{\begin{array}{l}
\partial_{t} u-\mathcal{H} \partial_{x}^{2} u-\partial_{x}^{3} u+\partial_{x}\left(u^{2}\right)=0, \quad t \in \mathbb{R} \\
u(x, 0)=\varphi(x), \quad \varphi \in H^{s}(\mathbb{T})
\end{array}\right.
$$

Here $\mathbb{T}=\mathbb{R} \backslash 2 \pi, \mathcal{H}$ is the Hilbert transform

$$
\mathcal{H} f(x)=\text { p.v. } \frac{1}{\pi} \int \frac{f(x-y)}{y} d y .
$$

The Benjamin equation physically characterizes the vertical displacement (bounded above and below by rigid horizontal planes) of the interface between a thin layer of fluid atop and a much thicker layer of higher density fluid ( $c f$. [1]). The more general form of (1.1) could be stated as

$$
\left\{\begin{array}{l}
\partial_{t} u+\alpha \mathcal{H} \partial_{x}^{2} u+\beta \partial_{x}^{3} u+\partial_{x}\left(u^{2}\right)=0, \quad \alpha, \beta, t \in \mathbb{R} \\
u(x, 0)=\varphi(x), \quad \varphi \in H^{s}(\mathbb{T})
\end{array}\right.
$$

As a KdV type equation, (1.2) models a large amount of physical quantities. If $\alpha \neq 0$, $\beta=0,(1.2)$ is the IVP of the Benjamin-Ono (BO) equation. The $\mathrm{BO}$ equation is a model for one-dimensional long waves in deep stratified fluids and is completely integrable. It is also a typical dispersive model for which the solution map could not be obtained by a Picard approach in $L^{2}$-based Sobolev spaces. For its well-posedness theory, see e.g. [2-5] and the references therein. If $(\alpha, \beta)=(0,-1),(1.2)$ agrees with the well-known IVP of KdV

\section{祭 Springer}

C 2015 Shi and Li; licensee Springer. This is an Open Access article distributed under the terms of the Creative Commons Attribution License (http://creativecommons.org/licenses/by/4.0), which permits unrestricted use, distribution, and reproduction in any medium, provided the original work is properly credited. 
equation. The history of the well-posedness for $\mathrm{KdV}$ equation is quite rich and the literature extensive. For the non-periodic case, see e.g. [6-11]. The periodic case can be found in [9]. For the local well-posedness of the dispersion generalized periodic KdV equation, see [12-14] for example. Some recent results in the context of long waves in deep stratified fluids can be found in [15-20].

If $\alpha \neq 0, \beta \neq 0$, then (1.2) is the IVP of the Benjamin equation. There is some wellknown work as regards the Benjamin type equations; see [21-23] for example. The wellposedness of the Benjamin equation in the non-periodic case were fully discussed by Chen et al. in their recent two papers [24, 25]. For the periodic case, there were less results for (1.2) than the non-periodic case. Only in [26], Linares obtained the well-posedness of (1.1) with initial value in $L^{2}$ space. In this paper, (1.1) will be taken as our model to study the IVP of the periodic Benjamin equation. We will prove that the local well-posedness result of Linares, valid for $s \geq 0$, can be extended to $s \geq-1 / 2$.

For the scaling argument, the following $\gamma$-periodic IVP for Benjamin equation should be considered:

$$
\left\{\begin{array}{l}
\partial_{t} u-\mathcal{H} \partial_{x}^{2} u-\partial_{x}^{3} u+\partial_{x}\left(u^{2}\right)=0, \quad t \in \mathbb{R} \\
u(x, 0)=\varphi(x), \quad \varphi \in H^{s}(0, \gamma)
\end{array}\right.
$$

Now, we formulate the main result of this paper as follows.

Theorem 1.1 For $s \geq-1 / 2, \gamma \lesssim 1$ and $\varphi \in H^{s}$, there exists $T=T\left(\|\varphi\|_{H^{s}}\right)>0$ such that (1.3) has a unique solution $u \in C\left([0, T], H^{s}\right) \cap Y^{s}$. The solution map $T: \varphi \rightarrow u$ is an analytic map on $H^{s}$.

The definitions of $X^{s}, Y^{s}$, and $H^{s}$ will be given in Section 2. To prove Theorem 1.1, the following bilinear estimates are crucial to our analysis.

Theorem 1.2 For $s \geq-1 / 2$ and $f, g \in X^{s}$, the following two bilinear estimates are true:

$$
\left(\frac{1}{\gamma} \sum_{k \in \dot{\mathbb{Z}} \backslash \gamma}|k|^{2 s} \int_{\mathbb{R}} \frac{\left|\hat{w}_{f g}(k, \lambda)\right|^{2}}{\langle\lambda+\phi(k)\rangle} d \lambda\right)^{1 / 2} \lesssim\|f\|_{X^{s}}\|g\|_{X^{s}}
$$

and

$$
\left(\frac{1}{\gamma} \sum_{k \in \dot{\mathbb{Z}} \gamma}|k|^{2 s}\left[\int_{\mathbb{R}} \frac{\left|\hat{w}_{f g}(k, \lambda)\right|}{\langle\lambda+\phi(k)\rangle} d \lambda\right]^{2}\right)^{1 / 2} \lesssim\|f\|_{X^{s}}\|g\|_{X^{s}},
$$

where $\hat{w}_{f g}(k, \lambda)=\frac{i}{\gamma^{2}} k \hat{f} *_{\gamma} \hat{g}(k, \lambda), \phi(k)=k^{3}-|k| k$.

Since Miura's transform does not work for the Benjamin equation, the way to set up the ill-posedness of $\mathrm{KdV}$ equation fails in dealing with that of the Benjamin equation. However, we can provide a counterexample to show that the bilinear estimates (1.4) and (1.5) fail for $s<-1 / 2$.

Theorem 1.3 For $s<-1 / 2$, (1.4) and (1.5) fail.

Here and in the following, for the sake of convenience, the abbreviations $\langle\xi\rangle=\left(1+|\xi|^{2}\right)^{1 / 2}$ for $\xi \in \mathbb{R}$ and $A \lesssim B=: A \leq C B$ are used, where $C$ is a positive constant and may change 
from line to line. Similarly, $A \ll B$ is used to represent $A \leq C^{-1} B$ and $A \sim B$ stands for $A \lesssim B \lesssim A$.

We end this section with the outline of this paper. In Section 2, some preliminaries which are essential to our proofs are collected. Section 3 contains the proofs of Theorem 1.1 and Theorem 1.2. In Section 4, Theorem 1.3 is proved by constructing a counterexample to verify that the bilinear estimates (1.4) and (1.5) fail for $s<-1 / 2$.

\section{Preliminaries}

We begin this section with some basic notations. The Fourier transform on $[0, \gamma]$ is defined as

$$
\hat{u}(k)=\frac{1}{\gamma} \int_{0}^{\gamma} u(x) \exp (-i k x) d x .
$$

The Fourier inversion formula is denoted by

$$
u(x)=\int \exp (2 \pi i k x) \hat{u}(k)(d k)_{\gamma}
$$

where $(d k)_{\gamma}$ is the normalized counting measure on $\mathbb{Z} \backslash \gamma$ and

$$
\int f(x)(d k)_{\gamma}=\frac{1}{\gamma} \sum_{k \in \mathbb{Z} \backslash \gamma} f(k)
$$

It is easy to check that the classical properties of the Fourier transform hold for the measure $(d k)_{\gamma}$. That is,

$$
\begin{aligned}
& \|u\|_{L^{2}([0, \gamma])}=\|\hat{u}\|_{L^{2}\left((d k)_{\gamma}\right)}, \\
& \widehat{u v}(k)=\hat{u} *_{\gamma} \hat{v}(k)=\int \hat{u}\left(k-k_{1}\right) \hat{v}\left(k_{1}\right)\left(d k_{1}\right)_{\gamma} .
\end{aligned}
$$

For more information as regards the $\gamma$-periodic Fourier transform, see [9] for example.

For $s \in \mathbb{R}$, the spatial Sobolev spaces are defined by

$$
H_{x}^{s}(0, \gamma):=\left\{u \in \mathcal{S}^{\prime}(\mathbb{T}):\|u\|_{H_{x}^{s}}=\left\|\langle\cdot\rangle^{s} \hat{u}\right\|_{L^{2}\left((d k)_{\gamma}\right)}<\infty\right\} .
$$

Let $P_{0}$ denote the mean operator

$$
P_{0} u:=\frac{1}{\gamma} \int_{0}^{\gamma} u d x
$$

or equivalently $P_{0} u=\hat{u}(0)$. The solutions of the Benjamin equation are mean-preserving, and it will be convenient to assume that

$$
\hat{u}(0)=\frac{1}{\gamma} \int_{0}^{\gamma} \varphi(x) d x=0
$$

which allows us to replace $k \in \mathbb{Z} \backslash \gamma$ with $k \in \dot{\mathbb{Z}} \backslash \gamma(\dot{\mathbb{Z}}=\mathbb{Z} \backslash\{0\})$. One can easily pass from the mean-zero case to the general mean case by the Galilean transformation $u(t, x) \mapsto$ 
$u\left(t, x-P_{0}(u) t\right)-P_{0}(u)$, which was introduced in [27]. $Y^{s}$ is the completion of functions that are Schwartz in time and $C^{\infty}$ in space with the following norm:

$$
\|u\|_{Y^{s}}=\|u\|_{X^{s}}+\left\|\langle k\rangle^{s} \hat{u}(k, \lambda)\right\|_{\left.L^{2}((d k))_{\gamma}\right) L^{1}(d \lambda)},
$$

where

$$
\|u\|_{X^{s}}=\left\|\langle k\rangle^{s}\langle\lambda+\phi(k)\rangle^{1 / 2} \hat{u}(k, \lambda)\right\|_{L^{2}\left((d k)_{\gamma} d \lambda\right)} .
$$

In fact, the study of the periodic Benjamin equation has been based on iteration in the space $X^{s}$, but this space barely fails to control the $L_{t}^{\infty} H_{x}^{s}$ norm. $Y^{s}$ is a slight modification of $X^{s}$ such that $\|u\|_{L_{t}^{\infty} H_{x}^{s}} \lesssim\|u\|_{Y^{s}}$.

The nonlinear part of the Benjamin equation is $u \partial_{x} u$, which can be written by a Fourier transform in frequency as

$$
k \sum_{k_{1} \in \dot{\mathbb{Z}} \gamma \gamma} \int_{\lambda_{1} \in \mathbb{R}} \hat{u}\left(k_{1}, \lambda_{1}\right) \hat{u}\left(k-k_{1}, \lambda-\lambda_{1}\right) d \lambda_{1} .
$$

During the argument, we mainly focus on the iteration of the two functions in (2.1), which were understood as two free Benjamin flows iterating with each other. The resonance function

$$
\begin{aligned}
R\left(k, k_{1}\right) & =\lambda+\phi(k)-\left(\lambda_{1}+\phi\left(k_{1}\right)\right)-\left(\lambda-\lambda_{1}+\phi\left(k-k_{1}\right)\right) \\
& =-k|k|+k_{1}\left|k_{1}\right|+\left(k-k_{1}\right)\left|k-k_{1}\right|+3 k k_{1}\left(k-k_{1}\right)
\end{aligned}
$$

gives a description of the set where two Benjamin flows interact. The resonance function is a very important concept in the analysis of the nonlinear dispersion equations. The following fundamental estimates for the resonance function can be proved easily.

Lemma 2.1 Let $\left|k k_{1}\left(k-k_{1}\right)\right| \neq 0, k, k_{1} \in \mathbb{Z} \backslash \gamma$ and $\gamma \lesssim 1$. Then

$$
\begin{aligned}
& \left|R\left(k, k_{1}\right)\right| \gtrsim\left|k k_{1}\left(k-k_{1}\right)\right|, \\
& |k|^{2} \lesssim\left|k k_{1}\left(k-k_{1}\right)\right| \lesssim\left(\max \left\{|k|,\left|k_{1}\right|\right\}\right)^{3} .
\end{aligned}
$$

Proof Equation (2.4) is obvious. The proof of (2.3) is a straightforward calculation with consideration of the following six cases following from [25]:

$$
\begin{aligned}
& G^{+++}=\left\{k-k_{1}>0, k>0, k_{1}>0\right\} ; \\
& G^{++-}=\left\{k-k_{1}>0, k>0, k_{1}<0\right\} ; \\
& G^{+--}=\left\{k-k_{1}>0, k<0, k_{1}<0\right\} ; \\
& G^{---}=\left\{k-k_{1}<0, k<0, k_{1}<0\right\} ; \\
& G^{-++}=\left\{k-k_{1}<0, k<0, k_{1}>0\right\} ; \\
& G^{-++}=\left\{k-k_{1}<0, k>0, k_{1}>0\right\} .
\end{aligned}
$$

The following fundamental estimates can be proved with a slight modification in $[14,28]$. 
Lemma 2.2 There exists $C>0$ such that for any $\varepsilon>0, \alpha \in \mathbb{R}$, and $0<\rho<1$,

$$
\begin{aligned}
& I(\alpha)=\int_{\mathbb{R}} \frac{d \beta}{(1+|\beta|)(1+|\alpha-\beta|)} \lesssim \frac{\log (2+|\alpha|)}{(1+|\alpha|)} \\
& I_{\rho}(\alpha)=\int_{\mathbb{R}} \frac{d \beta}{(1+|\beta|)^{\rho}(1+|\alpha-\beta|)} \lesssim \frac{1+\log (1+|\alpha|)}{(1+|\alpha|)^{\rho}} ; \\
& I^{\varepsilon}(\alpha)=\int_{\mathbb{R}} \frac{d \beta}{(1+|\beta|)^{1+\varepsilon}(1+|\alpha-\beta|)^{1+\varepsilon}} \lesssim \frac{1}{(1+|\alpha|)^{1+\varepsilon}} .
\end{aligned}
$$

Lemma 2.3 There exists $C>0$ such that for any $\rho>2 / 3, \gamma \lesssim 1, k, k_{1} \in \mathbb{Z} \backslash \gamma$, and $\lambda, \lambda_{1} \in \mathbb{R}$, the following estimates are true:

$$
\begin{aligned}
& \sum_{k_{1} \neq 0} \frac{\log \left(2+\left|\lambda+\phi\left(k_{1}\right)+\phi\left(k-k_{1}\right)\right|\right)}{\left(1+\left|\lambda+\phi\left(k_{1}\right)+\phi\left(k-k_{1}\right)\right|\right)} \leq C \gamma ; \\
& \sum_{k \neq 0} \frac{\log \left(2+\left|\lambda_{1}+\phi(k)-\phi\left(k-k_{1}\right)\right|\right)}{\left(1+\left|\lambda_{1}+\phi(k)-\phi\left(k-k_{1}\right)\right|\right)} \leq C \gamma ; \\
& \sum_{k \neq 0} \frac{\log \left(1+\left|\lambda_{1}+\phi(k)-\phi\left(k-k_{1}\right)\right|\right)}{\left(1+\left|\lambda_{1}+\phi(k)-\phi\left(k-k_{1}\right)\right|\right)^{\rho}} \leq C \gamma .
\end{aligned}
$$

Proof The proof of (2.8) is given first. The fact that, for any $\varepsilon>0$,

$$
\log (1+|a|) \lesssim C_{\varepsilon}(1+|a|)^{\varepsilon}
$$

gives the following estimate by taking $\varepsilon=1 / 6$ :

$$
\sum_{k_{1} \neq 0} \frac{1}{\left(1+\left|\lambda+\phi\left(k_{1}\right)+\phi\left(k-k_{1}\right)\right|\right)^{5 / 6}} \leq C .
$$

Let $\beta_{1}(k, \lambda), \beta_{2}(k, \lambda)$ be two complex roots of $\lambda+\phi\left(k_{1}\right)+\phi\left(k-k_{1}\right)$. In view of $\lambda+\phi\left(k_{1}\right)+$ $\phi\left(k-k_{1}\right)$ being a polynomial of degree 2 in the variable $k_{1},(2.11)$ can be rewritten as

$$
\sum_{k_{1} \neq 0} \frac{1}{\left(1+\left|k_{1}-\beta_{1}(k, \lambda)\right|\left|k_{1}-\beta_{2}(k, \lambda)\right|\right)^{5 / 6}} \leq C .
$$

Since

$$
\left|k_{1}-\beta_{j}(k, \lambda)\right| \geq\left|k_{1}-R_{j}(k, \lambda)\right|
$$

$\beta_{j}(k, \lambda)$ can be substituted by its real part $R_{j}(k, \lambda)$ for $j=1,2$. Set

$$
Z_{2}=\left\{k_{1}:\left|k_{1}-\beta_{j}(k, \lambda)\right| \leq 2, j=1,2\right\} .
$$

Then $Z_{2}$ has no more than $10 \gamma$ numbers $k_{1}$, therefore (2.12) can be controlled by

$$
10 \gamma+\sum_{\left|k_{1}-\beta_{j}(k, \lambda)\right|>2} \frac{1}{\left(1+\left|k_{1}-R_{1}(k, \lambda)\right|\left|k_{1}-R_{2}(k, \lambda)\right|\right)^{5 / 6}} .
$$


By the elementary inequality

$$
\left(1+a_{1}\right)\left(1+a_{2}\right) \leq 2\left(1+a_{1} a_{2}\right)
$$

for $a_{i} \geq 2, i=1,2$, it is sufficient to estimate

$$
\sum_{k_{1}} \frac{1}{\left(1+\left|k_{1}-R_{1}(k, \lambda)\right|\right)^{5 / 6}\left(1+\left|k_{1}-R_{2}(k, \lambda)\right|\right)^{5 / 6}} .
$$

And hence (2.11) follows by the Hölder inequality with $k_{1}$ in the above term.

The same conclusion can be drawn for (2.9) by replacing $k_{1}$ with $k$ in (2.8), while the proof of (2.10) can be handled in much the same way as that of (2.9) for $2(\rho-1 / 6)>1$.

In the following analysis, $\rho=5 / 6$ in (2.10) is always used. Next, we use the lower bound of the resonance function to recover the derivative on the nonlinear term $u \partial_{x} u$.

\section{Lemma 2.4 Let}

$$
Q_{s}=\frac{|k|^{2 s+2}\left|k_{1}\left(k-k_{1}\right)\right|^{-2 s}}{\sigma\left(\lambda, \lambda_{1}, k, k_{1}\right)}
$$

and

$$
Q_{s, r}=\frac{|k|^{2 s+2}\left|k_{1}\left(k-k_{1}\right)\right|^{-2 s}}{\sigma^{2(1-r)}\left(\lambda, \lambda_{1}, k, k_{1}\right)}
$$

where

$$
\sigma\left(\lambda, \lambda_{1}, k, k_{1}\right)=\max \left\{|\lambda+\phi(k)|,\left|\lambda_{1}+\phi\left(k_{1}\right)\right|,\left|\lambda-\lambda_{1}+\phi\left(k-k_{1}\right)\right|\right\}
$$

with $k k_{1}\left(k-k_{1}\right) \neq 0$. Then for $s \geq-1 / 2,0<r<1 / 4$, and $\gamma \lesssim 1$, we have

$$
Q_{s} \lesssim 1, \quad Q_{s, r} \lesssim \frac{1}{|n|^{2-4 r}} .
$$

Proof It can be confirmed easily by Lemma 2.1 that

$$
\sigma\left(\lambda, \lambda_{1}, k, k_{1}\right) \gtrsim\left|k k_{1}\left(k-k_{1}\right)\right| \text {. }
$$

Putting this lower estimate into $Q_{s}$ and $Q_{s, r}$, we can obtain

$$
Q_{s} \leq \frac{|k|^{2 s+2}\left|k_{1}\left(k-k_{1}\right)\right|^{-2 s}}{\left|k k_{1}\left(k-k_{1}\right)\right|} \lesssim \frac{|k|^{2 s+1}}{\left|k_{1}\left(k-k_{1}\right)\right|^{2 s+1}} \lesssim 1
$$

and

$$
Q_{s, r} \leq \frac{|k|^{2 s+2}\left|k_{1}\left(k-k_{1}\right)\right|^{-2 s}}{\left|k k_{1}\left(k-k_{1}\right)\right|^{2(1-r)}} \lesssim \frac{|k|^{2 s+1}}{|k|^{1-2 r}\left|k_{1}\left(k-k_{1}\right)\right|^{2 s+2(1-r)}} \lesssim \frac{1}{|k|^{2-4 r}},
$$

where (2.4) is used in the last estimate. 


\section{Proofs of Theorem 1.1 and Theorem 1.2}

For a function $f \in X^{s}$, set

$$
P_{f}(k, \lambda)=|k|^{s}\left\langle\lambda+\left.\phi(k)\right|^{1 / 2}|\hat{f}(k, \lambda)|\right.
$$

Then

$$
\begin{aligned}
& \|f\|_{X^{s}}=\left(\frac{1}{\gamma} \sum_{k \in \dot{\mathbb{Z}} / \gamma} \int_{\mathbb{R}}\left(P_{f}(k, \lambda)\right)^{2} d \lambda\right)^{1 / 2}=\left\|P_{f}(k, \lambda)\right\|_{\left.L^{2}((d k))_{\gamma} d \lambda\right)}, \\
& \left|\hat{w}_{f g}(k, \lambda)\right| \lesssim \frac{1}{\gamma}|k| \sum_{k_{1} \in \dot{\mathbb{Z}} \backslash \gamma} \int_{\mathbb{R}}\left|\hat{f}\left(k-k_{1}, \lambda-\lambda_{1}\right) \hat{g}\left(k_{1}, \lambda_{1}\right)\right| d \lambda_{1} .
\end{aligned}
$$

Since $\hat{u}(0)=0$, it is convenient to assume that $k k_{1}\left(k-k_{1}\right) \neq 0$.

We first prove Theorem 1.2. The integral in the left hand of (1.4) can be controlled by

$$
\frac{1}{\gamma} \sum_{k_{1}} \int_{\mathbb{R}} \frac{\left|k_{1}\left(k-k_{1}\right)\right|^{-s}|k|^{s+1} P_{f} P_{g} d \lambda_{1}}{(1+|\lambda+\phi(k)|)^{1 / 2}\left(1+\left|\lambda_{1}+\phi\left(k_{1}\right)\right|\right)^{1 / 2}\left(1+\left|\lambda-\lambda_{1}+\phi\left(k-k_{1}\right)\right|\right)^{1 / 2}},
$$

where $P_{f}:=P_{f}\left(k-k_{1}, \lambda-\lambda_{1}\right), P_{g}:=P_{g}\left(k_{1}, \lambda_{1}\right)$. Denote by

$$
Q\left(k, \lambda, k_{1}, \lambda_{1}\right)=\frac{|k|^{s+1}\left|k_{1}\left(k-k_{1}\right)\right|^{-s}}{\langle\lambda+\phi(k)\rangle^{1 / 2}\left\langle\lambda_{1}+\phi\left(k_{1}\right)\right\rangle^{1 / 2}\left\langle\lambda-\lambda_{1}+\phi\left(k-k_{1}\right)\right\rangle^{1 / 2}},
$$

then (1.4) equals

$$
\begin{aligned}
& {\left[\frac{1}{\gamma} \sum_{k} \int_{\mathbb{R}}\left(\sum_{k_{1}} \int_{\mathbb{R}} Q\left(k, \lambda, k_{1}, \lambda_{1}\right) P_{f} P_{g} d \lambda_{1}\right)^{2} d \lambda\right]^{1 / 2}} \\
& \quad \lesssim\left\|P_{f}(k, \lambda)\right\|_{L^{2}\left(\lambda,(d k)_{\gamma}\right)}\left\|P_{g}(k, \lambda)\right\|_{L^{2}\left(\lambda,(d k)_{\gamma}\right)} \\
& \quad=\|f\|_{X^{s}}\|g\|_{X^{s}} .
\end{aligned}
$$

Write

$$
A=\left\{\left(k, \lambda, k_{1}, \lambda_{1}\right):\left|\lambda-\lambda_{1}+\phi\left(k-k_{1}\right)\right| \leq\left|\lambda_{1}+\phi\left(k_{1}\right)\right|\right\},
$$

then by symmetry, the proof of (3.2) is reduced to the estimate

$$
\left[\frac{1}{\gamma} \sum_{k} \int_{\mathbb{R}}\left(\sum_{k_{1}} \int_{\mathbb{R}}\left(\chi_{A} Q\right)\left(k, \lambda, k_{1}, \lambda_{1}\right) P_{f} P_{g} d \lambda_{1}\right)^{2} d \lambda\right]^{1 / 2},
$$

which can be considered by two cases.

Case I. $\left|\lambda_{1}+\phi\left(k_{1}\right)\right| \leq|\lambda+\phi(k)|$. In this case, set $A$ is replaced by

$$
A_{\mathrm{I}}=\left\{\left|\lambda-\lambda_{1}+\phi\left(k-k_{1}\right)\right| \leq\left|\lambda_{1}+\phi\left(k_{1}\right)\right| \leq|\lambda+\phi(k)|\right\} .
$$


Cauchy-Schwarz's inequality allows us to control (3.3) by

$$
\left\|\left(\frac{1}{\gamma} \sum_{k_{1}} \int_{\mathbb{R}}\left(\chi_{A_{\mathrm{I}}} Q\right)^{2}\left(k, \lambda, k_{1}, \lambda_{1}\right) d \lambda_{1}\right)^{\frac{1}{2}}\left(\frac{1}{\gamma} \sum_{k_{1}} \int_{\mathbb{R}} P_{f}^{2} P_{g}^{2} d \lambda_{1}\right)^{\frac{1}{2}}\right\|_{L^{2}\left((d k)_{\gamma}\right) d \lambda} .
$$

We claim that there exists $C>0$ such that, for $s \geq-1 / 2$,

$$
S_{\mathrm{I}}^{2}=\sup _{k, \lambda} \frac{1}{\gamma} \sum_{k_{1}} \int_{\mathbb{R}}\left(\chi_{A_{\mathrm{I}}} Q\right)^{2}\left(k, \lambda, k_{1}, \lambda_{1}\right) d \lambda_{1} \lesssim C
$$

which gives

$$
(3.3) \leq S_{\mathrm{I}}\left\|P_{f}(k, \lambda)\right\|_{L^{2}\left((d k)_{\gamma}\right) d \lambda}\left\|P_{g}(k, \lambda)\right\|_{L^{2}\left((d k)_{\gamma}\right) d \lambda} \lesssim\|f\|_{X^{s}}\|g\|_{X^{s}}
$$

Indeed, the fact

$$
\langle\lambda+\phi(k)\rangle \geq \max \left\{\left\langle\lambda_{1}+\phi\left(k_{1}\right)\right\rangle,\left\langle\lambda-\lambda_{1}+\phi\left(k-k_{1}\right)\right\rangle\right\}
$$

allows us to write $Q^{2}$ as

$$
\begin{aligned}
Q^{2} & =\frac{|k|^{2 s+2}\left|k_{1}\left(k-k_{1}\right)\right|^{-2 s}}{\langle\lambda+\phi(k)\rangle} \frac{1}{\left\langle\lambda_{1}+\phi\left(k_{1}\right)\right\rangle\left\langle\lambda-\lambda_{1}+\phi\left(k-k_{1}\right)\right\rangle} \\
& \approx Q_{s} \frac{1}{\left\langle\lambda_{1}+\phi\left(k_{1}\right)\right\rangle\left\langle\lambda-\lambda_{1}+\phi\left(k-k_{1}\right)\right\rangle} .
\end{aligned}
$$

Lemma 2.4 with $s \geq-1 / 2,\left(k, \lambda, k_{1}, \lambda_{1}\right) \in A_{\text {I }}$ gives

$$
S_{\mathrm{I}}^{2} \lesssim \frac{1}{\gamma} \sup _{k, \lambda} \sum_{k_{1}} \int_{\mathbb{R}} \frac{d \lambda_{1}}{\left\langle\lambda_{1}+\phi\left(k_{1}\right)\right\rangle\left\langle\lambda-\lambda_{1}+\phi\left(k-k_{1}\right)\right\rangle}
$$

Let

$$
\beta=\lambda_{1}+\phi\left(k_{1}\right), \quad \alpha=\lambda+\phi\left(k_{1}\right)+\phi\left(k-k_{1}\right)=\tau-R\left(k, k_{1}\right), \quad \tau=\lambda+\phi(k) .
$$

Then (3.4) can be obtained by (2.5) and (2.8).

Case II. $|\lambda+\phi(k)|<\left|\lambda_{1}+\phi\left(k_{1}\right)\right|$. In this case, set $A$ is

$$
A_{\mathrm{II}}=\left\{\left|\lambda-\lambda_{1}+\phi\left(k-k_{1}\right)\right|<\left|\lambda_{1}+\phi\left(k_{1}\right)\right|,|\lambda+\phi(k)|<\left|\lambda_{1}+\phi\left(k_{1}\right)\right|\right\} .
$$

That is, we need to estimate the following term:

$$
\left\|\frac{1}{\gamma} \sum_{k_{1}} \int_{\mathbb{R}}\left(\chi_{A_{\mathrm{II}}} Q\right)\left(k, \lambda, k_{1}, \lambda_{1}\right) P_{f} P_{g} d \lambda_{1}\right\|_{L^{2}\left((d k)_{\gamma} d \lambda\right)} .
$$

By duality, (3.5) equals

$$
\sup _{\|d\|_{L^{2}((d k) \gamma d \lambda)}=1} \frac{1}{\gamma^{2}} \sum_{k, k_{1}} \int_{\mathbb{R}^{2}} d(k, \lambda)\left(\chi_{A_{\mathrm{II}}} Q\right)\left(k, \lambda, k_{1}, \lambda_{1}\right) P_{f} P_{g} d \lambda_{1} d \lambda
$$


Fubini's theorem and the Cauchy-Schwarz inequality can be used to control (3.6) by

$$
\begin{aligned}
& \sup _{\|d\|_{L^{2}((d k) \gamma d \lambda)}=1} \frac{1}{\gamma^{2}}\left(\sum_{k_{1}} \int_{\mathbb{R}}\left[\sum_{k} \int_{\mathbb{R}}\left(\chi_{A_{\mathrm{II}}} Q\right)^{2}\left(k, \lambda, k_{1}, \lambda_{1}\right) d \lambda\right]\right. \\
& \left.\times\left[\sum_{k} \int_{\mathbb{R}} d^{2}(k, \lambda) P_{f}^{2} d \lambda\right] d \lambda_{1}\right)^{1 / 2}\|g\|_{X^{s}}
\end{aligned}
$$

Write

$$
S_{\mathrm{II}}^{2}=\frac{1}{\gamma} \sup _{k_{1}, \lambda_{1}} \sum_{k} \int_{\mathbb{R}}\left(\chi_{A_{\mathrm{II}}} Q\right)^{2}\left(k, \lambda, k_{1}, \lambda_{1}\right) d \lambda
$$

Next, one only needs to show that, for $s \geq-1 / 2$,

$$
S_{\mathrm{II}}^{2}<\infty
$$

which is similar to the previous analysis of (3.4). For $\left(k, \lambda, k_{1}, \lambda_{1}\right) \in A_{\mathrm{II}}, Q^{2}$ could be written as

$$
\begin{aligned}
Q^{2} & =\frac{|k|^{2 s+2}\left|k_{1}\left(k-k_{1}\right)\right|^{-2 s}}{\left\langle\lambda_{1}+\phi\left(k_{1}\right)\right\rangle} \frac{1}{\langle\lambda+\phi(k)\rangle\left\langle\lambda-\lambda_{1}+\phi\left(k-k_{1}\right)\right\rangle} \\
& \approx Q_{s} \frac{1}{\langle\lambda+\phi(k)\rangle\left\langle\lambda-\lambda_{1}+\phi\left(k-k_{1}\right)\right\rangle} .
\end{aligned}
$$

Using Lemma 2.4, $S_{\mathrm{II}}^{2}$ can be estimated by

$$
S_{\mathrm{II}}^{2} \lesssim \frac{1}{\gamma} \sup _{k_{1}, \lambda_{1}} \sum_{k} \int_{\lambda} \frac{d \lambda}{\langle\lambda+\phi(k)\rangle\left\langle\lambda-\lambda_{1}+\phi\left(k-k_{1}\right)\right\rangle}
$$

Let

$$
\beta=\lambda+\phi(k), \quad \alpha=\lambda_{1}+\phi(k)-\phi\left(k-k_{1}\right)=\tau_{1}-R\left(k, k_{1}\right), \quad \tau_{1}=-\lambda_{1}-\phi\left(k_{1}\right) .
$$

Then (2.5) and (2.9) can be used to obtain (3.7). The proof of (1.4) is completed.

Next, we proceed the proof of Theorem 1.2 by showing (1.5). We first rewrite (1.5) as

$$
\left\|\frac{1}{\gamma} \int_{\mathbb{R}} \sum_{k_{1}} \int_{\mathbb{R}}\left(\chi_{A} Q\right)\left(\cdot, \lambda, k_{1}, \lambda_{1}\right) P_{f} P_{g} d \lambda_{1} d \lambda\right\|_{L^{2}\left((d k)_{\gamma}\right)} \lesssim\|f\|_{X^{s}}\|g\|_{X^{s}}
$$

where

$$
Q\left(k, \lambda, k_{1}, \lambda_{1}\right)=\frac{|k|^{s+1}\left|k_{1}\left(k-k_{1}\right)\right|^{-s}}{\langle\lambda+\phi(k)\rangle\left\langle\lambda_{1}+\phi\left(k_{1}\right)\right\rangle^{1 / 2}\left\langle\lambda-\lambda_{1}+\phi\left(k-k_{1}\right)\right\rangle^{1 / 2}} .
$$

As in the proof of (1.4), (1.5) can be considered by two cases.

Case I. $\left|\lambda_{1}+\phi\left(k_{1}\right)\right| \leq|\lambda+\phi(k)|$. In this case, the set $A$ is replaced by

$$
A_{\mathrm{I}}=\left\{\left|\lambda-\lambda_{1}+\phi\left(k-k_{1}\right)\right| \leq\left|\lambda_{1}+\phi\left(k_{1}\right)\right| \leq|\lambda+\phi(k)|\right\}
$$


By duality of $L^{2}\left((d k)_{\gamma}\right)$, one only needs to estimate

$$
\sup _{\|d\|_{L^{2}((d k) \gamma)}=1} \frac{1}{\gamma^{2}} \sum_{k} d(k) \int_{\mathbb{R}} \sum_{k_{1}} \int_{\mathbb{R}}\left(\chi_{A_{I}} Q\right)\left(k, \lambda, k_{1}, \lambda_{1}\right) P_{f} P_{g} d \lambda_{1} d \lambda,
$$

which can be bounded by

$$
\begin{aligned}
& \sup _{\|d\|_{L^{2}((d k) \gamma)}=1} \frac{1}{\gamma^{3 / 2}}\left[\sum_{k_{1}} \int_{\mathbb{R}}\left(\sum_{k} \int_{\mathbb{R}}\left(\chi_{A_{\mathrm{I}}} Q_{2}\right)^{2}\left(k, \lambda, k_{1}, \lambda_{1}\right) d \lambda\right)\right. \\
& \left.\times\left(\sum_{k} \int_{\mathbb{R}} d^{2}(k) P_{f}^{2} d \lambda\right) d \lambda_{1}\right]^{1 / 2}\|g\|_{X^{s}}
\end{aligned}
$$

by Cauchy-Schwarz's inequality. Therefore, it is sufficient to show that for $s \geq-1 / 2$,

$$
S_{\mathrm{I}}^{2}=\sup _{k_{1}} \frac{1}{\gamma} \sum_{k} \int_{\mathbb{R}} \int_{\mathbb{R}}\left(\chi_{A_{\mathrm{I}}} Q\right)^{2}\left(k, \lambda, k_{1}, \lambda_{1}\right) d \lambda d \lambda_{1} \lesssim C .
$$

For some $0<r<1 / 4$,

$$
S_{\mathrm{I}}^{2}=\sup _{k_{1}} \frac{1}{\gamma} \sum_{k} \int_{\mathbb{R}} \int_{\mathbb{R}} \frac{1}{\langle\lambda+\phi(k)\rangle^{2 r}}\left(\chi_{A_{\mathrm{I}}} Q_{r}\right)^{2}\left(k, \lambda, k_{1}, \lambda_{1}\right) d \lambda d \lambda_{1},
$$

where

$$
\begin{aligned}
Q_{r}^{2} & =\frac{|k|^{2 s+2}\left|k_{1}\left(k-k_{1}\right)\right|^{-2 s}}{\langle\lambda+\phi(k)\rangle^{2(1-r)}} \cdot \frac{1}{\left\langle\lambda_{1}+\phi\left(k_{1}\right)\right\rangle\left\langle\lambda-\lambda_{1}+\phi\left(k-k_{1}\right)\right\rangle} \\
& =Q_{s, r} \frac{1}{\left\langle\lambda_{1}+\phi\left(k_{1}\right)\right\rangle\left\langle\lambda-\lambda_{1}+\phi\left(k-k_{1}\right)\right\rangle} .
\end{aligned}
$$

By Lemma 2.4, $S_{\text {I }}^{2}$ could be controlled by

$$
\sup _{k_{1}} \frac{1}{\gamma} \sum_{k} \int_{\mathbb{R}} \int_{\mathbb{R}} \frac{1}{|k|^{2-4 r}} \cdot \frac{d \lambda_{1} d \lambda}{\left\langle\lambda_{1}+\phi\left(k_{1}\right)\right\rangle^{1+r}\left\langle\lambda-\lambda_{1}+\phi\left(k-k_{1}\right)\right\rangle^{1+r}} .
$$

Applying (2.7) with $\alpha=\lambda+\phi\left(k_{1}\right)+\phi\left(k-k_{1}\right)$, one has

$$
\begin{gathered}
\int_{\mathbb{R}} \frac{d \lambda_{1}}{\left\langle\lambda_{1}+\phi\left(k_{1}\right)\right\rangle^{1+r}\left\langle\lambda-\lambda_{1}+\phi\left(k-k_{1}\right)\right\rangle^{1+r}} \\
\quad \lesssim \frac{1}{\left(1+\left|\lambda+\phi\left(k_{1}\right)+\phi\left(k-k_{1}\right)\right|\right)^{1+r}} ;
\end{gathered}
$$

hence

$$
S_{I}^{2} \lesssim \sup _{k_{1}} \frac{1}{\gamma} \sum_{k} \frac{1}{|k|^{2-4 r}} \int_{\mathbb{R}} \frac{1}{\left(1+\left|\lambda+\phi\left(k_{1}\right)+\phi\left(k-k_{1}\right)\right|\right)^{1+r}} d \lambda,
$$

and finally $S_{\mathrm{I}}^{2}<\infty$ for $r<1 / 4$.

Case II. $|\lambda+\phi(k)|<\left|\lambda_{1}+\phi\left(k_{1}\right)\right|$. In this case, $A$ is replaced by

$$
A_{\mathrm{II}}=\left\{\left|\lambda-\lambda_{1}+\phi\left(k-k_{1}\right)\right|<\left|\lambda_{1}+\phi\left(k_{1}\right)\right|,|\lambda+\phi(k)|<\left|\lambda_{1}+\phi\left(k_{1}\right)\right|\right\} .
$$


Write

$$
1+|\lambda+\phi(k)|=(1+|\lambda+\phi(k)|)^{r}(1+|\lambda+\phi(k)|)^{1-r},
$$

where $1 / 2<r<1$. As in Case I, let

$$
Q_{r}=\frac{|k|^{s+1}\left|k_{1}\left(k-k_{1}\right)\right|^{-s}}{\left\langle\lambda_{1}+\phi\left(k_{1}\right)\right\rangle^{1 / 2}\langle\lambda+\phi(k)\rangle^{1-r}\left\langle\lambda-\lambda_{1}+\phi\left(\left(k-k_{1}\right)\right)\right\rangle^{1 / 2}} .
$$

Then it suffices to estimate

$$
\left[\frac{1}{\gamma} \sum_{k}\left(\int_{\mathbb{R}} \frac{1}{\gamma} \sum_{k_{1}} \int_{\mathbb{R}} \frac{1}{\langle\lambda+\phi(k)\rangle^{r}}\left(\chi_{A_{\mathrm{II}}} Q_{r}\right)\left(k, \lambda, k_{1}, \lambda_{1}\right) P_{f} P_{g} d \lambda_{1} d \lambda\right)^{2}\right]^{1 / 2} .
$$

Applying the Cauchy-Schwarz inequality in $\lambda$, (3.8) can be bounded by

$$
\left[\frac{1}{\gamma} \sum_{k}\left(\int_{\mathbb{R}} \frac{d \lambda}{\langle\lambda+\phi(k)\rangle^{2 r}}\right)\left(\int_{\mathbb{R}}\left\{\frac{P_{f} P_{g}}{\gamma} \sum_{k_{1}} \int_{\mathbb{R}}\left(\chi_{A_{\mathrm{II}}} Q_{r}\right)\left(k, \lambda, k_{1}, \lambda_{1}\right) d \lambda_{1}\right\}^{2} d \lambda\right)\right]^{1 / 2} .
$$

Since $2 r>1,(3.8)$ is dominated by

$$
\left[\frac{1}{\gamma} \sum_{k} \int_{\mathbb{R}}\left\{\frac{1}{\gamma} \sum_{k_{1}} \int_{\mathbb{R}}\left(\chi_{A_{\mathrm{II}}} Q_{r}\right)\left(k, \lambda, k_{1}, \lambda_{1}\right) P_{f} P_{g} d \lambda_{1}\right\}^{2} d \lambda\right]^{1 / 2} .
$$

By the definition of the $L_{\left((d k)_{\gamma} d \lambda\right)}^{2}$-norm, (3.8) can be further controlled by

$$
\sup _{\|d\|_{\left.L(d k)_{\gamma} d \lambda\right)}^{2}=1} \frac{1}{\gamma} \sum_{k} \int_{\mathbb{R}} d(k, \lambda) \frac{1}{\gamma} \sum_{k_{1}} \int_{\mathbb{R}}\left(\chi_{A_{\mathrm{II}}} Q_{r}\right)\left(k, \lambda, k_{1}, \lambda_{1}\right) P_{f} P_{g} d \lambda_{1} d \lambda .
$$

By the Cauchy-Schwarz inequality, we can obtain the following estimate for (3.9):

$$
\begin{aligned}
& \sup _{\|d\|_{\left.L(d k)_{\gamma} d \lambda\right)=1}^{2}}\left[\frac{1}{\gamma} \sum_{k_{1}} \int_{\mathbb{R}}\left(\frac{1}{\gamma} \sum_{k} \int_{\mathbb{R}}\left(\chi_{A_{1}} Q_{r}\right)^{2}\left(k, \lambda, k_{1}, \lambda_{1}\right) d \lambda\right)\right. \\
& \left.\times\left(\frac{1}{\gamma} \sum_{k} \int_{\mathbb{R}} d^{2}(k, \lambda) P_{f}^{2} d \lambda\right) d \lambda_{1}\right]^{1 / 2}\|g\|_{X^{s}} .
\end{aligned}
$$

For $s \geq-1 / 2$, we claim that

$$
S_{\mathrm{II}}^{2}=\sup _{k_{1}, \lambda_{1}} \frac{1}{\gamma} \sum_{k} \int_{\mathbb{R}}\left(\chi_{A_{\mathrm{I}}} Q_{r}\right)^{2}\left(k, \lambda, k_{1}, \lambda_{1}\right) d \lambda<\infty
$$

In fact, by Lemma 2.4, we have

$$
S_{\mathrm{II}}^{2}=\sup _{k_{1}, \lambda_{1}} \frac{1}{\gamma} \sum_{k} \int_{\mathbb{R}} \frac{d \lambda}{\langle\lambda+\phi(k)\rangle^{2(1-r)}\left\langle\lambda-\lambda_{1}+\phi\left(k-k_{1}\right)\right\rangle} .
$$

Applying (2.6) with $\rho=2(1-r), \beta=\lambda+\phi(k)$, and $\alpha=\lambda_{1}+\phi(k)-\phi\left(k-k_{1}\right)$ for some $1 / 2<$ $r<1$, we get $S_{\text {II }}^{2}<\infty$ as a result of (2.10). Theorem 1.2 has thus been proved. 
Having disposed of the proof for Theorem 1.2, we can now prove Theorem 1.1. Taking Fourier transform with respect to $x$ in (1.3) gives

$$
\partial_{t} \hat{u}(k, t)-i k|k| \hat{u}(k, t)+i k^{3} \hat{u}(k, t)=-\hat{w}(k, t), \quad \hat{u}(k, 0)=\hat{\varphi}(k),
$$

where $\hat{w}(k, t)=\widehat{u \partial_{x} u}(k, t)$. The $Y^{s}$ norm cannot be calculated directly on the equation since the function has no integrability in time. A cutting off according to time is necessary. Let $\psi(t)$ be a bump function with support in $\{|t|<1\}$ and $\psi(t)=1$ for $|t|<1 / 2$. Then the following can be shown:

$$
\begin{aligned}
\psi(t) u(x, t)= & \frac{1}{\gamma} \psi(t) \sum_{k \in \dot{\mathbb{Z}} / \gamma} \hat{\varphi}(k) e^{i(k x-t \phi(k))} \\
& +\frac{i}{\gamma^{2}} \sum_{n=1}^{\infty} \frac{i^{n} t^{n}}{n !} \psi(t) \sum_{k \in \dot{\mathbb{Z}} / \gamma} e^{i(k x-t \phi(k))} \\
& \times \int_{-\infty}^{\infty} \psi(\lambda+\phi(k))(\lambda+\phi(k))^{n-1} \hat{w}(k, \lambda) d \lambda \\
& +\frac{i}{(\gamma)^{2}} \psi(t) \sum_{k \in \dot{\mathbb{Z}} / \gamma} e^{i(k x)} \int_{-\infty}^{\infty} \frac{1-\psi(\lambda+\phi(k))}{\lambda+\phi(k)} e^{i \lambda t} \hat{w}(k, \lambda) d \lambda \\
& -\frac{i}{(\gamma)^{2}} \psi(t) \sum_{k \in \dot{\mathbb{Z}} / \gamma} e^{i(k x-t \phi(k))} \int_{-\infty}^{\infty} \frac{1-\psi(\lambda+\phi(k))}{\lambda+\phi(k)} \hat{w}(k, \lambda) d \lambda
\end{aligned}
$$

where $\hat{w}(k, \lambda)=\frac{i}{\gamma^{2}} k \hat{u} * \hat{u}(k, \lambda)$. We adopt the idea in [28] dealing with the KdV equation here to prove Theorem 1.1. Let $T$ denote the map defined by the above expressions of $\psi(t) u(x, t)$. Then the task is to show that the map $u \mapsto T u$ is contracted in the space $Y^{s}$. To do this, we need the following estimates.

Theorem 3.1 Let $s \geq-1 / 2$. Then there exist constants $C=C(\psi)>0$ such that for all $u, v \in$ $Y^{s}$,

$$
\begin{aligned}
& \|T u\|_{Y^{s}} \leq C\left(\|u\|_{Y^{s}}^{2}+\|\varphi\|_{H^{s}}\right) \\
& \|T u-T v\|_{Y^{s}} \leq C\left(\|u+v\|_{Y^{s}}\|u-v\|_{Y^{s}}\right) .
\end{aligned}
$$

A straightforward application of Theorem 3.1 can produce the following proposition.

Proposition 3.2 Let $C=C(\psi)$ be the constant appearing in Theorem 3.1. If $\|\varphi\|_{H^{s}} \leq$ $2 /\left(9 C^{2}\right)$, then $T$ is a contraction in the closed ball $B(0,1 /(3 C))$ of $Y^{s}$.

Therefore, the proof of Theorem 1.1 for sufficiently small data $\left(\|\varphi\|_{H^{s}} \leq 2 /\left(9 c^{2}\right)\right)$ follows from Proposition 3.2.

Next, we give the sketch of the proof for Theorem 3.1. Computing the $Y^{s}$-norm of the terms in (3.11), the following estimate can be obtained:

$$
\|T u\|_{Y^{s}} \lesssim\|\varphi\|_{H^{s}}+\left(\|w\|_{X^{s,-\frac{1}{2}}}+\left(\frac{1}{\gamma} \sum_{k \in \dot{Z} / \gamma}|k|^{2 s}\left[\int_{\mathbb{R}} \frac{|\hat{w}(k, \lambda)|}{\langle\lambda+(k)\rangle} d \lambda\right]^{2}\right)^{1 / 2}\right)
$$


Then (3.11) follows from (3.13) and the bilinear estimates in Theorem 1.2 with $f=g=u$. Noticing that $T u-T v$ is a dispersion Benjamin equation with nonlinear term $u \partial_{x} u-v \partial_{x} v$ with initial data $\varphi=0,(3.12)$ can be proved by the bilinear estimates with $f=u+v$ and $g=u-v$.

\section{Proof of Theorem 1.3}

As explained in the above sections, the method used to prove the local well-posedness of the Benjamin equation is based on the bilinear estimates (1.4) and (1.5). In this section, a counterexample will be given to show that (1.4) and (1.5) are not true for $s<-1 / 2$ inspired by some ideas from [11].

For any $F=P_{f}(k, \lambda) \in L^{2}\left((d k)_{\gamma} d \lambda\right)$, the bilinear form $\mathfrak{B}_{s}(F, F)$ can be defined by

$$
\frac{k^{s+1}}{\langle\lambda+\phi(k)\rangle^{1 / 2}} \sum_{k_{1} \neq 0, k_{1} \neq k} \int_{-\infty}^{\infty} \frac{\left(k_{1}\left(k-k_{1}\right)\right)^{-s} F\left(k_{1}, \lambda_{1}\right) F\left(k-k_{1}, \lambda-\lambda_{1}\right) d \lambda_{1}}{\left\langle\lambda_{1}+\phi\left(k_{1}\right)\right\rangle^{1 / 2}\left\langle\lambda-\lambda_{1}+\phi\left(k-k_{1}\right)\right\rangle^{1 / 2}} .
$$

Then (1.4) equals

$$
\left\|\mathfrak{B}_{S}(F, F)\right\|_{L^{2}\left((d k)_{\gamma} d \lambda\right)} \lesssim c\|F\|_{L^{2}\left((d k)_{\gamma} d \lambda\right)}^{2}
$$

where $s \geq-1 / 2$. A counterexample will be given next to show that (4.1) fails for $s<-1 / 2$. Choosing

$$
F=a_{k} \chi_{1}(\lambda+\phi(k))
$$

where

$$
\chi_{b}(\lambda+\phi(k))= \begin{cases}1, & |\lambda+\phi(k)| \leq b \\ 0, & \text { elsewhere }\end{cases}
$$

and

$$
a_{k}= \begin{cases}1, & k=N, 1-N \\ 0, & \text { elsewhere }\end{cases}
$$

then $\mathfrak{B}_{s}(F, F)(k, \lambda)$ at $k=1$ can be evaluated as

$$
\mathfrak{B}_{s}(F, F)(1, \lambda) \approx \frac{1}{(1+|\lambda+\phi(k)|)^{1 / 2}} \cdot \frac{1}{N^{2 s}} .
$$

For those $\lambda$ which are in a unit size interval of $\lambda_{1}$, the following can be shown:

$$
\left|\lambda_{1}+\phi(N)\right| \leq 1, \quad\left|\lambda-\lambda_{1}+\phi(1-N)\right| \leq 1 .
$$

Moreover, the lower bound estimate on the resonance function implies

$$
|\lambda+\phi(1)| \gtrsim N^{2} .
$$


Hence, for any $N \in \mathbb{N}$,

$$
\frac{1}{N} \cdot \frac{1}{N^{2 s}} \leq C
$$

Therefore, $s \geq-1 / 2$. The same function can be used to show that (1.5) fails for $s<-1 / 2$. This completes the proof of Theorem 1.3.

\section{Competing interests}

The authors declare that they have no competing interests.

\section{Authors' contributions}

All authors contributed equally to the writing of this paper. All authors read and approved the final manuscript.

\section{Author details}

${ }^{1}$ Department of Mathematics, Linyi University, Linyi, 276005, P.R. China. ${ }^{2}$ Laboratory of Mathematics and Complex Systems, Ministry of Education, Beijing, 100875, P.R. China. ${ }^{3}$ School of Mathematical Sciences, Beijing Normal University, Beijing, 100875, P.R. China.

\section{Acknowledgements}

The authors are thankful to the honorable reviewers for their valuable suggestions and comments, which improved the paper. This work was partially supported by National Natural Science Foundation of China (Grant Nos. 11171026, $11271175,11301249,11301250)$, National Natural Science Foundation of Shandong Province (Grant No. ZR2012AQ026) the Applied Mathematics Enhancement Program of Linyi University, and also the Program for Changjiang Scholars and Innovative Research Team in University.

Received: 14 January 2015 Accepted: 27 March 2015 Published online: 04 April 2015

\section{References}

1. Benjamin, B: A new kind of solitary wave. J. Fluid Mech. 245, 401-411 (1992)

2. Guo, Z, Huo, Z: The well-posedness of KdV-Benjamin-Ono equation in low regularity spaces. J. Am. Math. Soc. 20, 753-798 (2007)

3. Ionescu, A, Kenig, C: Global well-posedness of the Benjamin-Ono equation. J. Am. Math. Soc. 20, 753-798 (2007)

4. Koch, $H$, Tzvetkov, N: On the local well-posedness of the Benjamin-Ono equation in $H^{5}(\mathbb{R})$. Int. Math. Res. Not. 2003, 1449-1464 (2003)

5. Tao, T: Global well-posedness of the Benjamin-Ono equation in $H^{1}(\mathbb{R})$. J. Hyperbolic Differ. Equ. 1, 27-49 (2004)

6. Bourgain, J: Fourier transform restriction phenomena for certain lattice subsets and applications to nonlinear evolution equations. I. Schrödinger equations. Geom. Funct. Anal. 3, 107-156 (1993)

7. Bourgain, J: Fourier transform restriction phenomena for certain lattice subsets and applications to nonlinear evolution equations. II. The KdV-equation. Geom. Funct. Anal. 3, 209-262 (1993)

8. Christ, M, Colliander, J, Tao, T: Asymptotics, frequency modulation and low regularity ill-posedness for canonical defocusing equations. Am. J. Math. 125, 1235-1293 (2003)

9. Colliander, J, Keel, M, Staffilani, G, Takaoka, H, Tao, T: Sharp global well-posedness for KdV and modified KdV on $\mathbb{R}$ and T. J. Am. Math. Soc. 16, 705-749 (2003)

10. Guo, Z: Global well-posedness of Korteweg-de Vries equation in $H^{-3 / 4}(\mathbb{R})$. J. Math. Pures Appl. 91, 583-597 (2009)

11. Kenig, C, Ponce, G, Vega, L: On the ill-posedness of some canonical dispersive equations. Duke Math. J. 106, 617-633 (2001)

12. Chen, W, Guo, Z: Global well-posedness and I-method for the fifth-order Korteweg-de Vries equation. J. Anal. Math. $114,121-156(2011)$

13. Chen, W, Li, J, Miao, C, Wu, J: Low regularity solutions of two fifth-order KdV type equations. J. Anal. Math. 107 221-238 (2009)

14. Li, J, Shi, S: Local well-posedness for the dispersion generalized periodic KdV equation. J. Math. Anal. Appl. 379 706-718 (2011)

15. Alias, A, Grimshaw, RHJ, Khusnutdinova, KR: Coupled Ostrovsky equations for internal waves in a shear flow. Phys. Fluids 26, 126603 (2014)

16. Alias, A, Grimshaw, RHJ, Khusnutdinova, KR: On strongly interacting internal waves in a rotating ocean and coupled Ostrovsky equations. Chaos 23, 013126 (2013)

17. Khusnutdinova, KR, Samsonov, AM: Fission of a longitudinal strain solitary wave in a delaminated bar. Phys. Rev. E 77, 066603 (2008)

18. Ruggieri, M, Speciale, MP: New exact solutions for a coupled KdV-like model. J. Phys. Conf. Ser. 482, 012036 (2014)

19. Ruggieri, M, Speciale, MP: Similarity reduction and closed form solutions for a model derived from two-layer fluids. Adv. Differ. Equ. 2013, 355 (2013)

20. Ruggieri, M, Speciale, MP: Conservation laws for a model derived from two layer-fluids. J. Phys. Conf. Ser. 482,012037 (2014)

21. Angulo, J: Existence and stability of solitary wave solution of the Benjamin equation. J. Differ. Equ. 152, 136-159 (1999)

22. Chen, $\mathrm{H}$, Bona, J: Existence and asymptotic properties of solitary-wave solutions of Benjamin-type equations. Adv. Differ. Equ. 3, 51-84 (1998)

23. Zhao, M, Yang, $X$, Zhang, L: Averaging of the $3 D$ non-autonomous Benjamin-Bona-Mahony equation with singularly oscillating forces. Bound. Value Probl. 2013, 111 (2013). doi:10.1186/1687-2770-2013-111 
24. Chen, W, Guo, Z, Xiao, J: Sharp well-posedness of the Benjamin equation. Nonlinear Anal. 74, 6209-6230 (2011)

25. Chen, W, Xiao, J: A sharp bilinear estimate for the Bougain-type space with application to the Benjamin equation. Commun. Partial Differ. Equ. 35, 1739-1762 (2010)

26. Linares, F: $L^{2}$ Global well-posedness of the initial value problem associated to the Benjamin equation. J. Differ. Equ. 152, 377-393 (1999)

27. Himonas, A, Misiolek, G: Well-posedness of the Cauchy problem for a shallow water equation on the circle. J. Differ. Equ. 161, 479-495 (2000)

28. Gorsky, J, Himonas, A: Well-posendness of KdV with higher dispersion. Math. Comput. Simul. 80, 173-183 (2009)

Submit your manuscript to a SpringerOpen ${ }^{\circ}$ journal and benefit from:

- Convenient online submission

- Rigorous peer review

- Immediate publication on acceptance

- Open access: articles freely available online

- High visibility within the field

Retaining the copyright to your article

Submit your next manuscript at $\boldsymbol{s p r i n g e r o p e n . c o m ~}$ 